\title{
Evaluating the Effectiveness of Small-Group Training in Teaching Medical Students Integral Clinical Eye Examination Skills
}

\author{
John Y. Lee, BA ${ }^{1, *}$ Ryan A. Gallo, BA ${ }^{1, *(1)} \quad$ Chrisfouad R. Alabiad, MD ${ }^{1}$ \\ ${ }^{1}$ Department of Ophthalmology, Bascom Palmer Eye Institute, \\ University of Miami Miller School of Medicine, Miami, Florida \\ Address for correspondence Chrisfouad Alabiad, MD, Department of \\ Ophthalmology, Bascom Palmer Eye Institute, University of Miami \\ Miller School of Medicine, 900 NW 17th Street, Miami, FL 33136 \\ J Acad Ophthalmol 2020;12:e79-e86. \\ (e-mail: CAlabiad@med.miami.edu).
}

\begin{abstract}
Keywords

- eye examination

- direct ophthalmoscopy

- medical student education

- small-group training

- confidence level

Objective The aim of this study is to evaluate the effectiveness of an interactive, small-group ophthalmology clinical training session by assessing medical students' self-confidence with eye examination skills and long-term retention of direct ophthalmoscopy skills.

Methods The second-year medical students participated in a one-time small-group clinical training session that taught essential components of the eye examination. Students reported their confidence with each component in pre- and postsession surveys. Eight months later, direct ophthalmoscopy skills were reassessed by having students visualize the optic nerves of standardized patients and identify the matching optic nerve photograph in a multiple-choice quiz.

Results Among 197 second-year medical students who participated in the training session, 172 students completed the presession survey (87.3\% response rate) and 108 students completed the postsession survey (54.8\% response rate). Following the training session, students reported increased self-confidence $(p<0.01)$ overall. A total of 107 (107/108; 99.1\%) students reported that they visualized the optic nerve head, and 80 out of 85 (94.1\%) students stated that they preferred the PanOptic ophthalmoscope over the traditional direct ophthalmoscope. Students reported greater selfconfidence using the PanOptic ophthalmoscope $(p<0.01)$. In the 8-month follow-up assessment, 42 medical students $(42 / 197 ; 21.3 \%)$ completed the exercise. A total of 41 (41/42; 97.6\%) students stated that they saw the optic nerve with the PanOptic ophthalmoscope; 24 (24/42; 57.1\%) students identified the correct optic nerve image using the PanOptic ophthalmoscope on a standardized patient; 14 (14/42; 33.3\%) students stated that they saw the optic nerve with the traditional direct ophthalmoscope; and 4 (4/42; 9.1\%) students from the same cohort identified the correct optic nerve image with the traditional direct ophthalmoscope on a standardized patient.

Conclusion Our comprehensive, one-time eye examination skills training session seeks to prepare students to incorporate these skills in future patient care. Students' overall confidence improved in each aspect of the eye examination that was covered. A
\end{abstract}

The authors contributed equally to this work.

received

January 4, 2020

accepted

April 13, 2020
DOI https://doi.org/

10.1055/s-0040-1712175. ISSN 2475-4757.
Copyright $\odot 2020$ by Thieme Medical Publishers, Inc., 333 Seventh Avenue, New York, NY 10001, USA. Tel: +1(212) 760-0888.
License terms

()(1) $\Theta \circledast$ 
follow-up assessment on students' direct ophthalmoscopy skills suggests that the PanOptic ophthalmoscope allows for superior skills retention as compared with the traditional direct ophthalmoscope. We believe that the PanOptic ophthalmoscope should be further integrated into medical education and clinical practice.

The Association of University Professors in Ophthalmology (AUPO) and International Council of Ophthalmology (ICO) Taskforce have standards for ophthalmic proficiency expected of all graduating medical students. These standards highlight several fundamental components of the eye examination, including proficiency in measuring visual acuity; detecting abnormal eye movements, pupillary responses, and ocular surface abnormalities; and performing direct ophthalmoscopy to evaluate the optic nerve head and fundus. $^{1,2}$ Despite these recommendations, both medical students and practicing nonophthalmologists infrequently perform a basic eye examination or do so poorly. ${ }^{3,4}$

While several barriers may contribute to this problem, one of the most apparent obstacles is the difficulty of teaching procedural skills in a medical student curriculum. Especially in a technically heavy field like ophthalmology, proper training with unique instruments is needed for one to evaluate symptoms, dictate initial management, or even refer patients with some of the most basic ophthalmic problems..$^{5-7}$ Considerable investments in equipment, instructors, and time are required to train medical students adequately. Nonetheless, it is critical for medical school graduates to learn an accurate and thorough eye examination, as several sight- and life-threatening conditions can be discerned by the proper implementation of these skills. ${ }^{3,8}$

In response to this need, we designed and implemented an ophthalmology clinical skills training session that was integrated with a preexisting 1 -week long ophthalmology preclinical course. In this study, we evaluate the effectiveness and utility of this one-time skills workshop, which may serve as a practical teaching model. We assess students' reported self-confidence with eye examination skills, gauge their preferences for the traditional direct or PanOptic ophthalmoscope, and explore long-term retention of fundoscopy skills in an objective assessment on standardized patients. We used this information to suggest changes to nonophthalmology fundoscopy standard practices.

\section{Methods}

\section{Study Population}

Participants were second-year medical students at the University of Miami Miller School of Medicine. Inclusion criteria required participation in the preclinical ophthalmology course, completion of a presession quiz, and attendance at the small-group skills training session. The University of Miami Institutional Review Board determined that this study meets the criteria for an exemption as described in Federal Regulation 45 CFR 46.104.

\section{Overview of Protocol}

This small-group training session was integrated with the second-year medical students' 1-week, preclinical ophthalmology course that was held in July 2019. The training was conducted in a 90-minute session midway through the course. Each group was led primarily by fourth-year medical students entering ophthalmology along with ophthalmology residents, fellows, and faculty from Bascom Palmer Eye Institute (BPEI). Fourth-year medical students were selected to be trainers by an attending ophthalmologist prior to the training session after having demonstrated proficiency in performing and teaching a comprehensive eye examination. Pre- and postsurveys on students' confidence in performing each part of the eye examination were administered. A cohort of students in our combined MD/MPH dual degree program that underwent end of year clinical competency exercises in March 2020 (8 months after the initial session) were assessed on their direct ophthalmoscopy skills using both the PanOptic ophthalmoscope and the traditional direct ophthalmoscope.

\section{Presession}

Medical students viewed presession instructional videos and completed an accompanying quiz. Students also completed a survey asking them to report their self-confidence in each aspect of the eye examination according to a 6-point Likert scale.

\section{Small-Group Training}

The second-year medical students were divided into 24 groups of eight to nine students. Groups were primarily led by fourth-year medical students who were trained and selected by an ophthalmology faculty member (C. R. A.). BPEI residents, fellows, and attendings led some groups and were "floating" instructors. The training session lasted 90 minutes. Each session began with 5 minutes of standardized instruction on how the training was organized.

The training sequence consisted of key components of the eye examination (-Fig. 1). The training sequence was split into two portions. In one portion, 40 minutes were allotted for training in external eye inspection, visual acuity, extraocular motility assessment, confrontation visual fields assessment, and intraocular pressure (IOP) measurement. In the other portion, 40 minutes were allotted for training in pupillary light reflex exam, corneal staining, and fundoscopic examination via direct ophthalmoscopy. Half of the groups started with one portion of the training, while the second half started with the other due to equipment limitations. Fluorescein sodium sterile ophthalmic strips $0.6 \mathrm{mg}$ (Akron, Lake Forest, IL) were used. Students were trained with the 

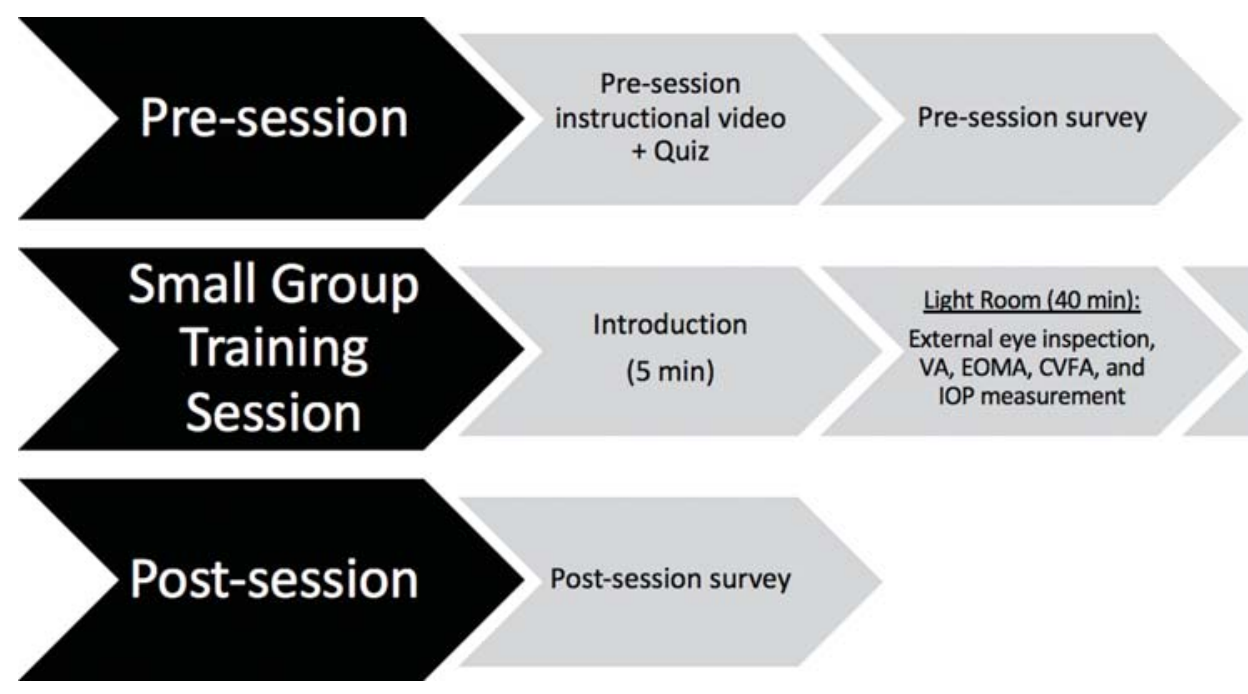

Fig. 1 Training sequence flowsheet. CVFA, confrontational visual field assessment; EOMA, extraocular motility assessment; IOP, intraocular pressure; VA, visual acuity.

Tono-Pen tonometer (Reichert Technologies, Depew, NY) for IOP measurement. Students were trained with traditional direct ophthalmoscopes and PanOptic ophthalmoscopes with and without an iExaminer adapter (Welch Allyn, Skaneateles Falls, NY).

Students practiced administering these elements of the eye examination on each other in pairs. Students were taught how to properly calibrate and use the Tono-Pen tonometer for measuring IOP. For direct ophthalmoscopy training, eye models provided by Welch Allyn were used when needed. Students were given equal time to practice on each other with traditional direct ophthalmoscopes and PanOptic ophthalmoscopes.

\section{Postsession}

The final 10 minutes of the session were dedicated to feedback. Students who completed the presession survey and attended the small-group training were asked to complete a postsession survey that collected information on their confidence with each element of the eye examination that was reviewed. They were also queried regarding which instrument they preferred for learning direct ophthalmoscopy. A 6point Likert scale was used again.

\section{Long-Term Follow-Up}

Optic nerve photographs of two standardized patients were taken at the BPEI Imaging Center. Separate multiple-choice quizzes were created for each standardized patient. Quizzes contained five options: four optic nerve photographs and an "unable to observe the optic nerve" option. Students who previously completed the training session 8 months prior were instructed to visualize a standardized patient's fundus with a traditional direct ophthalmoscope and another standardized patient's fundus with a PanOptic ophthalmoscope. Each standardized patient had pupillary diameter measured in dim light to be approximately $4 \mathrm{~mm}$. Students then completed the quiz for each patient immediately following fundus examination. This follow-up was held in March 2020 during their year-end clinic competency exercises.

\section{Statistics}

The paired Student's t-test was used to analyze the difference between pre- and postsession survey responses. The independent Student's $t$-test was used to compare preferences between the traditional direct and PanOptic ophthalmoscopes. All analyses were performed with consultation from the University of Miami Data Services team.

\section{Results}

Of the 197 second-year medical students who participated in our clinical skills training session, 172 students ( $87.3 \%$ response rate) completed the presession survey and 108 students (54.8\% response rate) completed the postsession survey. Participating students reported increased confidence in all components of the eye examination that were reviewed ( 2.80 vs. 4.52 , $p<0.01$ ), reflected by a change from "uncomfortable/neutral" to "extremely comfortable/comfortable." They reported the greatest absolute change in mean confidence with fluorescein staining of the cornea following the training session ( 0.67 vs. $4.31, p<0.001$ ). Students also reported increased confidence with observing the optic nerve with the PanOptic ophthalmoscope (1.21 vs. $4.48, p<0.001)$ and measuring IOP (1.62 vs. $4.25, p<0.001)$. Students reported the smallest absolute change in mean confidence with the pupillary exam after the training session (3.77 vs. $4.61, p<0.001$; - Fig. 2 ).

Overall, students were satisfied with the training session and perceived ophthalmology clinical skills to be important in their future medical careers. Most students were "extremely satisfied/satisfied" (mean score of 4.71) with the training that they received. A total of 107 out of 108 (99.1\%) students reported that they visualized the optic nerve with either the traditional direct or PanOptic ophthalmoscope. Students "strongly agreed/agreed" (mean score of 4.64) that fundamental eye examination skills will be beneficial for the care of their future patients and that they would "absolutely/occasionally" (mean score of 4.59) use their improved eye examination skills in their clinical practice ( - Table $\mathbf{1}$ ). 


\section{Mean Student Confidence Level}

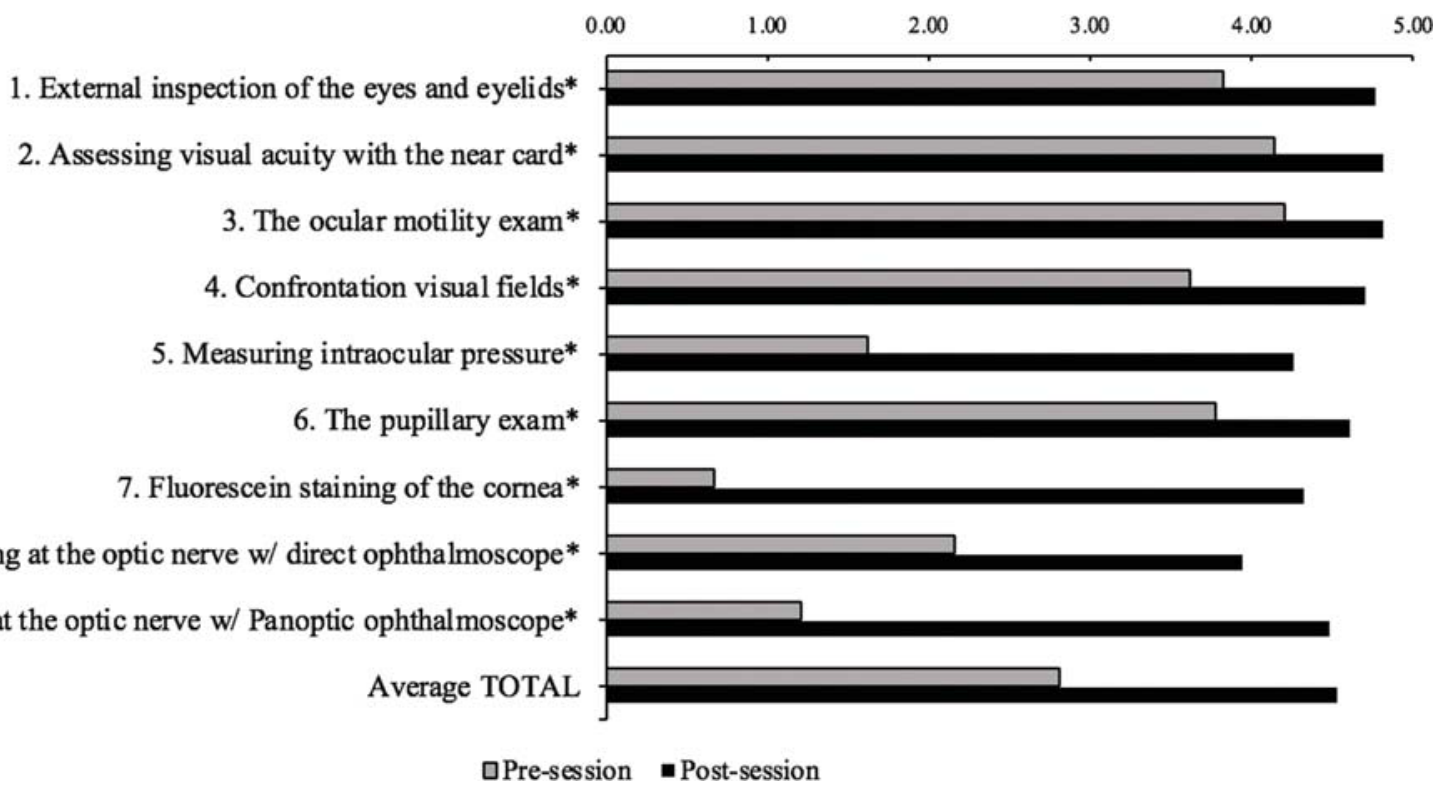

Fig. 2 Graphic representation of the mean student self-confidence levels in individual components of the eye examination using a paired onetailed Student's $t$-test. A 6-point Likert scale was used: (0) never tried, (1) very uncomfortable, (2) uncomfortable, (3) neutral, (4) comfortable, and (5) extremely comfortable. *denotes significance of $p$-value $<0.01$.

Table 1 Student perception of the small-group ophthalmology clinical skills training session

\begin{tabular}{|l|l|l|}
\hline Statement/question & $\begin{array}{l}\text { Mean } \\
\text { presession } \\
\text { response } \\
\text { (SD) }\end{array}$ & $\begin{array}{l}\text { Mean } \\
\text { postsession } \\
\text { response } \\
\text { (SD) }\end{array}$ \\
\hline $\begin{array}{l}\text { (a) A working knowledge of } \\
\text { the fundamentals of the } \\
\text { eye exam is beneficial } \\
\text { for the care of my } \\
\text { patients }(n=172)\end{array}$ & $4.64( \pm 0.55)$ & $4.59( \pm 0.58)$ \\
\hline $\begin{array}{l}\text { (b) Will skills today help } \\
\text { in future practice? } \\
\text { ( } n=108)\end{array}$ & $\begin{array}{l}\text { (c) Satisfaction with } \\
\text { today's session }(n=108)\end{array}$ & $4.71( \pm 0.67)$ \\
\hline Question & Yes & No \\
\hline $\begin{array}{l}\text { Did you see the optic } \\
\text { nerve today? }(n=108)\end{array}$ & 107 & 1 \\
\hline
\end{tabular}

Abbreviation: SD, standard deviation.

Students were asked about their opinion on the questions listed above:

(a) 1 = strongly disagree, 2 = disagree, $3=$ neutral, 4 = agree,

$5=$ strongly agree.

(b) $1=$ not at all, $2=$ possibly, $3=$ neutral, $4=$ occasionally, $5=$ absolutely.

(c) 1 = very unsatisfied, $2=$ unsatisfied, $3=$ neutral, $4=$ satisfied, $5=$ extremely satisfied.

Students also reported a clear preference for the PanOptic ophthalmoscope. Before the session, most students stated that they were more confident using the traditional direct ophthalmoscope than the PanOptic ophthalmoscope (2.30 vs. $1.16, p<0.001)$. However, after training with both instruments, the vast majority of students expressed greater confidence for visualizing the fundus and optic nerve head with the PanOptic ophthalmoscope (4.49 vs. 3.93, $p<0.001$ ). A total of 80 out of 85 students $(94.1 \%)$ preferred the PanOptic ophthalmoscope over the traditional direct ophthalmoscope (-Table 2 ).

A subset of the second-year class (MD/MPH dual degree students) that completed end of year competency exercises in March 2020 were assessed on their direct ophthalmoscopy skills using both the PanOptic ophthalmoscope and the traditional direct ophthalmoscope. These students did not have a formal review session of this exam technique since the initial session 8 months prior. Based on the multiple-choice quiz results following fundus examination on standardized patients, students demonstrated greater accuracy and ease with the PanOptic ophthalmoscope than with the traditional direct ophthalmoscope. With the PanOptic ophthalmoscope, $24(24 / 42 ; 57.1 \%)$ students identified the correct fundus image, $17(17 / 42 ; 40.5 \%)$ students selected an incorrect image, and $1(1 / 42 ; 2.4 \%)$ student could not find the optic nerve. With the traditional direct ophthalmoscope, 4 (4/42; 9.1\%) students identified the correct fundus image, $10(10 / 42 ; 23.8 \%)$ students selected the incorrect image, and 28 students (28/42; $66.7 \%$ ) could not find the optic nerve ( - Table 3 ).

\section{Discussion}

The Importance of Clinical Ophthalmology Skills Training Conducting an accurate and thorough eye examination is critical to the diagnoses of several sight- and life-threatening medical conditions. ${ }^{3,8}$ As gatekeepers to managed care, primary care physicians must be proficient in basic ophthalmology skills to detect signs of emergent vision loss, screen for retinal degenerative disease, and evaluate systemic and microvascular 
Table 2 Traditional direct ophthalmoscope versus PanOptic ophthalmoscope

\begin{tabular}{|c|c|c|c|c|c|c|}
\hline & & $\begin{array}{l}\text { Traditional } \\
\text { direct }\end{array}$ & PanOptic & Difference & $\begin{array}{l}\text { Fold } \\
\text { difference }\end{array}$ & $p$-Value \\
\hline \multirow[t]{2}{*}{ (a) } & $\begin{array}{l}\text { Mean presession confidence level (SD) } \\
n=172\end{array}$ & $2.16( \pm 1.16)$ & $1.21( \pm 1.37)$ & -0.95 & 0.51 & 7.09E-08 \\
\hline & $\begin{array}{l}\text { Mean postsession confidence level (SD) } \\
n=108\end{array}$ & $3.93( \pm 1.02)$ & $4.49( \pm 0.64)$ & 0.55 & 1.14 & $2.91 \mathrm{E}-06$ \\
\hline (b) & $\begin{array}{l}\text { Which did you find easier to use? } \\
\text { (number of students) }\end{array}$ & 5 & 80 & & & \\
\hline
\end{tabular}

Abbreviation: SD, standard deviation.

(a) Mean confidence levels between the traditional direct ophthalmoscope and PanOptic ophthalmoscope are compared using an independent onetailed Student's $t$-test. Students completed a survey to reflect their respective confidence levels $(0=$ never tried, $1=$ very uncomfortable,

$2=$ uncomfortable, 3 = neutral, $4=$ comfortable, 5 = extremely comfortable). Significant differences in confidence levels are reflected between the traditional direct and PanOptic ophthalmoscopes groups as noted in the pre- and postsession groups. Difference column denotes numeric change relative to PanOptic ophthalmoscope mean confidence level. adenotes $p$-value $<0.001$.

(b) Number of responses from students who preferred the traditional direct versus PanOptic ophthalmoscope are noted.

Table 3 Long-term retention of ophthalmoscopy skills with the traditional direct ophthalmoscope and PanOptic ophthalmoscope

\begin{tabular}{|l|l|l|l|}
\hline & $\mathbf{n = 4 2}$ & $\begin{array}{l}\text { Traditional } \\
\text { direct }\end{array}$ & PanOptic \\
\hline (a) & Correct responses (\%) & $4(9.5)$ & $24(57.1)$ \\
\hline & Incorrect responses (\%) & $10(23.8)$ & $17(40.5)$ \\
\hline & $\begin{array}{l}\text { Did not visualize } \\
\text { optic nerve (\%) }\end{array}$ & $28(66.7)$ & $1(2.4)$ \\
\hline (b) & $\begin{array}{l}\text { Students who visualized } \\
\text { optic nerve (\%) }\end{array}$ & $14(33.3)$ & $41(97.6)$ \\
\hline
\end{tabular}

(a) Number of students who correctly, incorrectly, or could not visualize the optic nerve in a multiple-choice quiz following examination of standardized patients.

(b) Number of students who affirm visualization of the optic nerve following fundus assessment of standardized patient.

disease (hypertension, diabetes, etc.). ${ }^{7,9}$ Emergency medicine physicians commonly perform instrument-based components of the eye examination, such as tonometry, fluorescein staining, and ophthalmoscopy, to assess for ocular trauma and acute retinopathy. ${ }^{10}$ A well-performed eye examination can reveal key diagnostic and prognostic findings of systemic diseases that are managed among many specialties.

There is a growing consensus that medical school graduates and nonophthalmology providers should be competent in key elements of the eye examination. The Association of American Medical Colleges, AUPO, the American Academy of Ophthalmology, and the ICO emphasize that medical students, at minimum, should be able to perform a basic eye examination with fundoscopy and describe their observations proficiently. ${ }^{1,11,12}$ Medical students should also be able to visualize the red reflex, the retina, and optic disc; assess the optic disc for cupping, color, contour, margins, vessels, and edema; and recognize changes associated with glaucoma and macular degeneration. ${ }^{1}$

Given that many unique instruments and imaging modalities are used to validate symptoms and reach diagnoses, substantial hands-on time is necessary for medical students to gain adequate knowledge, comfort, and confidence with these technical skills. However, ophthalmology education has become deprioritized in many medical school curriculums. Dedicated ophthalmology mean curriculum hours and mandated clinical teaching steadily declined in the past several years. ${ }^{13}$ As a result, many medical school graduates are inadequately trained and lack confidence in their ability to perform these important clinical skills upon entering residency training. ${ }^{5-7}$

It is reassuring to note that our medical students share the same sentiment for the importance of ophthalmology clinical skills in the future care of their patients (-Table 1 ). While students understand the gravity with which these skills can affect patient care, it is the utmost duty of medical educators to provide the time, resources, and comprehensive training for their students to obtain the knowledge and skills that they seek.

\section{Reflections and Evaluation of Our Small-Group Clinical Training Session}

Our one-time, small-group training session was effective in teaching our medical students fundamental components of the eye examination in a concise 90-minute session. In all components of the eye examination that were reviewed, students' confidence improved ( - Fig. 2). The greatest increase in reported confidence occurred with fluorescein staining of the cornea. This most likely occurred because students had the least amount of prior exposure to this skill. Low presession confidence levels directly correlate with the degree of unfamiliarity with both the instrument and technique, and the presession confidence was lowest in this component of the eye examination (mean score of 0.67 , "very uncomfortable/never tried"). Other components of the eye examination with low presession confidence levels (mean score $<2.00$ ) and hence lower degrees of familiarity, include observing the optic nerve head with the PanOptic ophthalmoscope and measuring IOP (mean score of 1.21 and 1.62, respectively).

Certain presession confidence levels are higher than expected because some medical students are exposed to ophthalmology clinical skills through our Department of Outreach and Community Services Health Fairs. These community health 
fairs include an ophthalmology station, where students conduct visual acuity screening, frequency doubling technology visual field testing, tonometry, and corneal pachymetry. Medical students attend a mandatory 30-minute skills session before each health fair to review these eye examination skills. They do not receive training in direct ophthalmoscopy as only physicians perform the fundus examination at the health fairs. Another reason for higher presession confidence levels than expected may be explained by a noncomprehensive understanding of an examination component. For example, a proper external inspection of the eyes and eyelids includes several different components such as noting eyelid lesions, proptosis, eyelid malpositions to name a few. Students may have felt confident in their perception of a thorough external eye examination without realizing that they may have omitted other important components of this particular examination. Hence, students could have falsely reported a higher level of confidence than what should have been attributed.

While the educational benefits of our ophthalmology clinical training session are reflected by our responses from second-year medical students, upperclassman medical students (primarily fourth-year students) who were smallgroup trainers also reaped the benefits of solidifying their own skills and further developing their capacity as medical educators. The "see one, do one, teach one" teaching methodology has been commonly used among medical trainees to gain proficiency in procedural skills and techniques. ${ }^{14}$ This adage reflects the traditional method of procedural teaching, where a trainee-after adequately observing a procedure-is expected to perform this procedure and eventually teach another trainee how to do the same. This teaching model is reflected in our clinical skills training design, where our upperclassmen trainers at one point observed, performed, and now teach these examination skills to lowerclassmen trainees. The capacity to teach another individual to perform a skill reflects the highest order of proficiency. By utilizing skilled upperclassmen to train underclassmen in our training session, we helped the upperclassmen advance their technical skills and develop the next generation of future trainers as part of a continual learning cycle.

\section{Traditional Direct versus PanOptic Ophthalmoscopy}

Of all the eye examination components in our study, students reported the lowest postsession confidence level (mean score of 3.93, "comfortable/neutral") in observing the optic nerve head with the traditional direct ophthalmoscope (-Fig. 2). We suspect that this due to the technical difficulties, many face with using this instrument. While there is a consensus that medical students and primary care providers should be proficient in using an ophthalmoscope, there is debate over how much proficiency is required. With a viewing field of 5 to 10 n degrees and a $15 \times$ magnification, the direct ophthalmoscope makes it challenging to obtain a proper visualization of the optic nerve head, not to mention regions away from the nerve. ${ }^{15}$ Evidence suggests that the traditional direct ophthalmoscope, even when used by ophthalmologists, is inadequate in identifying common retinal abnormalities. In a study comparing the traditional direct ophthalmoscope and gold standard (seven field fundus photography), direct ophthalmoscope examinations by comprehensive ophthalmologists and retina specialists agreed with the gold standard in only 52 and $70 \%$ of cases, respectively. $^{16}$

The alternatives, multifield fundus photography or indirect ophthalmoscopy, are either much costlier or difficult to carry out in a nonophthalmology practice. However, the PanOptic ophthalmoscope demonstrates promise as an alternative to the traditional direct ophthalmoscope. The PanOptic ophthalmoscope is a "direct" ophthalmoscope but has a wider field of view (25 degrees) and is more user-friendly than the traditional direct ophthalmoscope. The vast majority of our medical students preferred the PanOptic ophthalmoscope $(80 / 85 ; 94.1 \%)$ compared with the traditional direct ophthalmoscope. Students provided several comments regarding their strong preference for the PanOptic ophthalmoscope, including its larger field of view, easier maneuverability, stability, easier focus, further distance from patient, and clearer visualization of vessels and optic nerve head.

Students also exhibited superior retention of technical fundoscopy skills with the PanOptic ophthalmoscope in a long-term objective assessment on standardized patients (-Table 3). While most students (24/42; 57.1\%) identified the correct optic nerve image with the PanOptic ophthalmoscope, $66.7 \%$ (28/41) of the students could not even visualize the optic nerve with the traditional direct ophthalmoscope. Since a majority of students do not retain their skills with the traditional direct ophthalmoscope, teaching fundoscopy with this instrument may be less effective or may require more time in dedicated training. Students will be unable to assess crucial ophthalmic emergencies or identify essential findings associated with commonly managed diseases in their future clinical practices. Since skills retention with the PanOptic ophthalmoscope is better, focus in future sessions can be devoted to reviewing important clinical evaluation skills and pathology. Furthermore, given the greater ease the PanOptic ophthalmoscope affords, physicians who do not regularly perform ophthalmic exams may be inclined to do so more often. For these reasons, we believe that the PanOptic ophthalmoscope should replace the traditional direction ophthalmoscope not only in the medical education setting but also in nonophthalmologists' clinical practices.

\section{Long-Term Retention of Ophthalmoscopy Skills}

Students were not given the opportunity to review or practice with either ophthalmoscope before the follow-up assessment. They were markedly more accurate and comfortable using the PanOptic ophthalmoscope than the traditional direct ophthalmoscope (-Table 3 ). Our results also demonstrate that competency in technical skill does not necessarily correlate with clinical competency. Apart from the differences in student accuracy between the two ophthalmoscopes, there was a large discrepancy between subjective student responses affirming visualization of the optic nerve and the objective assessment on standardized 
patients. In the follow-up student cohort, 41 (41/42; 97.6\%) students visualized the optic nerve with the PanOptic ophthalmoscope, demonstrating excellent retention of technical capability. Yet, only $24(24 / 41 ; 58.5 \%)$ of these students identified the correct optic nerve image.

While the majority of these students were accurate with the PanOptic ophthalmoscope, the discrepancy in clinical competency is concerning. One obvious explanation for this discrepancy is the extended 8-month duration with which most students did not have any ophthalmology exposure, resulting in students forgetting key features of a baseline fundus assessment (cup, color, contour, margins, and vessels). Another contributing factor may be the differences in magnification between the ophthalmoscope view and optic nerve images in the multiple-choice quiz. The traditional ophthalmoscope view is considerably more magnified, requiring a bit of maneuvering to see the entire nerve and visual memory to compare what they see to the quiz image options which show the entire nerve in one photo; thus, students might not have evaluated enough necessary features to select the matching image. There is always the possibility that students simply guessed without actually visualizing the optic nerve on the standardized patients even though there was an option to select for those who could not visualize it. Despite these contributing factors, the conclusion remains that clinical assessment skills must be better integrated and reviewed with technical instruction.

\section{Limitations and Future Directions}

This study only evaluated a subset of medical students' clinical competency objectively and focused on self-reported confidence levels in performing each component of the eye examination for the majority of students. While perceived skill and self-confidence do not necessarily reflect actual skill proficiency, we believe that these factors are essential in mediating improved skills in future clinical training. Incorporating a comprehensive objective evaluation with a trained evaluator for all aspects of the eye examination would demonstrate both immediately after and several months after the session will provide a more accurate assessment of training success and skill retention.

Our study also did not evaluate students' ability to discern ocular pathology. The utility of learning examination skills is marginal without the ability to translate these skills to identifying pathology. Incorporating clinical scenarios through simulation or standardized patients may help reinforce the pathology that students learn in their concurrent ophthalmology preclinical course.

Other limitations of our study are the lower response rate of the postsession survey and the low student participation in the long-term follow-up exercise on standardized patients. These limitations may have influenced postsession results interpretation. The response rate was lower because the postsession survey was optional and did not accompany a mandatory quiz unlike the presession survey. While MD/MPH dual degree students were assessed during their end of year clinical competency exercises, extenuating circumstances forced our medical school to suspend all clinical and standardized patient activities for the remainder of the academic year. As a result, end of year clinical competency exercises scheduled for MD track students did not take place. However, this limited dataset is still valuable as it includes students who did not have a formal review session of direct ophthalmoscopy and who have a variety of different clinical interests.

Our study shows that students who participated in our course had an overall improvement in their comfort and confidence with the eye examination. Our course structure is succinct and sustainable given the small-group, peer-led format. This session can be incorporated into other medical institutions without major disruptions to their curriculums and may be able to better prepare their medical school students to identify basic ophthalmologic disease and emergencies.

Our study also reveals students' overwhelming preference for the PanOptic ophthalmoscope. We believe that the PanOptic ophthalmoscope should be further integrated into medical education and clinical practice. Given the dwindling hours dedicated to ophthalmology education, it would be more effective to train students with a tool that is easier to use and enables improved skills retention. However, these ophthalmoscopes are not widely available in most medical schools, clinical practices, or hospitals and may otherwise be economically burdensome. A major hurdle is challenging the norm of using the traditional direct ophthalmoscope. We believe that a shift in the standard of direct funduscopic visualization in the primary care setting is warranted and should be proposed for discussion. A study that collects data on the frequency with which primary care providers use the traditional direct ophthalmoscope in their clinical practice may shine light on the utility of this instrument and provide further impetus for change to other instruments like the PanOptic ophthalmoscope.

\section{Ethical Approval}

The University of Miami Institutional Review Board determined that this study meets the criteria for an exemption as described in Federal Regulation 45 CFR 46.104.

\section{Funding}

The Bascom Palmer Eye Institute is supported by the National Institutes of Health Center Core (grant number: P30EY014801) and a Research to Prevent Blindness Unrestricted Grant (New York, NY). J.Y.L. is supported by a Research to Prevent Blindness Medical Student Fellowship.

\section{Conflict of Interest}

None declared.

\section{Acknowledgments}

We thank M. Suits and Welch Allyn for graciously lending us PanOptic ophthalmoscopes, iExaminer adaptors, and eye simulators. R.K. Lee for providing us Tono-Pen tonometers. P. Mendez and A. Maurer for direct ophthalmoscopes. Lastly, we thank all the upperclassman medical 
students of the University of Miami Miller School of Medicine and the residents, fellows, and faculty of the Bascom Palmer Eye Institute who helped design and lead the training sessions.

\section{References}

1 International Task Force on Opthalmic Education of Medical Students; International Council of Opthalmology. Principles and guidelines of a curriculum for ophthalmic education of medical students. Klin Monatsbl Augenheilkd 2006;223(Suppl 5):S1-S19

2 Graubart EB, Waxman EL, Forster SH, et al. Ophthalmology objectives for medical students: revisiting what every graduating medical student should know. Ophthalmology 2018;125(12):1842-1843

3 Morad Y, Barkana Y, Avni I, Kozer E. Fundus anomalies: what the pediatrician's eye can't see. Int J Qual Health Care 2004;16(05): 363-365

4 Roberts E, Morgan R, King D, Clerkin L. Funduscopy: a forgotten art? Postgrad Med J 1999;75(883):282-284

5 Promes SB, Chudgar SM, Grochowski CO, et al. Gaps in procedural experience and competency in medical school graduates. Acad Emerg Med 2009;16(Suppl 2):S58-S62

6 Quillen DA, Harper RA, Haik BG. Medical student education in ophthalmology: crisis and opportunity. Ophthalmology 2005; 112(11):1867-1868

7 Stern GA; The Association of University Professors of Ophthalmology Education Committee. Teaching ophthalmology to primary care physicians. Arch Ophthalmol 1995;113(06):722-724
8 Hitchings RA. Visual disability and the elderly. BMJ 1989;298 (6681):1126-1127

9 Jacobs DS. Teaching doctors about the eye: trends in the education of medical students and primary care residents. Surv Ophthalmol 1998;42(04):383-389

10 Druck J, Valley MA, Lowenstein SR. Procedural skills training during emergency medicine residency: are we teaching the right things? West J Emerg Med 2009;10(03):152-156

11 Mottow-Lippa L. Ophthalmology in the medical school curriculum: reestablishing our value and effecting change. Ophthalmology 2009;116(07):1235-1236, 1236.e1

12 Corbett EC. Recommendations for clinical skills curricula for undergraduate medical education. Association of American Medical Colleges Task Force on the Preclerkship Clinical Skills Education of Medical Students 2008; Available at: stfm.org/ media/1363/clinicalskills_oct09qxdpdf.pdf. Accessed August 14, 2019

13 Shah M, Knoch D, Waxman E. The state of ophthalmology medical student education in the United States and Canada, 2012 through 2013. Ophthalmology 2014;121(06):1160-1163

14 Kotsis SV, Chung KC. Application of the "see one, do one, teach one" concept in surgical training. Plast Reconstr Surg 2013;131 (05):1194-1201

15 Benbassat J, Polak BC, Javitt JC. Objectives of teaching direct ophthalmoscopy to medical students. Acta Ophthalmol 2012;90 (06):503-507

16 Sussman EJ, Tsiaras WG, Soper KA. Diagnosis of diabetic eye disease. JAMA 1982;247(23):3231-3234 